\title{
Defined purge gas composition stabilizes molten nitrate salt - Experimental prove and thermodynamic calculations
}

\author{
Veronika Anna Sötz ${ }^{\mathrm{a}^{*}}$, Alexander Bonk $^{\mathrm{b}}$, Julian Steinbrecher ${ }^{\mathrm{b}}$ and Thomas Bauer ${ }^{\mathrm{a}}$ \\ a Institute of Engineering Thermodynamics, German Aerospace Center (DLR), Linder Höhe, 51147 Köln, Germany. \\ b Institute of Engineering Thermodynamics, German Aerospace Center (DLR), Pfaffenwaldring 38-40, 70569 Stuttgart, Germany. \\ *Corresponding author. Contact: +49 2203601 3606, Veronika.Soetz@dlr.de.
}

\section{$\underline{\text { Abstract }}$}

Thermal energy storage systems are integrated in concentrating solar power plants to improve the flexibility of the electricity generation. Commonly, the liquid storage material Solar Salt, a nitrate salt mixture, is applied to transport and store solar thermal energy. The lifetime and the temperature range of Solar Salt in the storage units are restricted by decomposition reactions of the material. Oxide ion formation is one of the fundamental issues. So far, it has not been proven if oxide ion formation can be prevented by addition of gaseous reaction products to the gas atmosphere. Also, a reliable reaction equation for the oxide ion formation is missing. In the presented experiments, molten salt at 600 and $620{ }^{\circ} \mathrm{C}$ is purged with a gas mixture of nitrogen, oxygen, and nitrous gases. Post-analysis of salt samples reveals stabilizing effects, depending on the purge gas compositions. Chemical equilibrium of the oxide ion forming reaction is demonstrated. It is proven that oxide ion formation can be controlled and suppressed. Reaction equations are evaluated and selected in order to quantify the reaction thermodynamics. The results contribute to recommendations for operating conditions and gas handling in storage systems of solar thermal power plants, which finally ensure reliable and constant material properties for extended lifetime and high temperatures.

Keywords: Thermal energy storage, Solar Salt, Salt decomposition, Oxide ion formation, Chemical equilibrium, Reaction thermodynamics

\section{$1 \quad$ Introduction}

\subsection{Technological background}

The concentrating solar power (CSP) technology transforms the solar irradiation to solar thermal energy, which is finally used for electricity generation. Large-scale and commercial CSP plants exist in several countries. Prospectively, the technology is considered to gain importance due to the transition to renewable energies. An energy storage unit adds flexibility to CSP plants, making electricity generation adjustable to the electricity market. Also, a storage unit enables continuous electricity supply during the night or in cloudy weather. For the CSP technology, thermal energy storage with a nitrate salt mixture commonly known as Solar Salt is the state-of-the-art storage type with a significant market. Those storage units belong to the class of sensible heat storage, because the material is used exclusively in its liquid state at different temperature levels. (Mehos et al., 2017; Tian and Zhao, 2013)

Sensible heat storage with Solar Salt has many advantages. The material features excellent pumpability due to its low viscosity (Janz et al., 1979; Janz et al., 1972; Jin et al., 2016), which is not the case for alternatively considered solid storage materials. It is applicable at ambient pressure thanks to its low vapor pressure (Glazov et al., 2002). Compared to halide salts which are also discussed for 